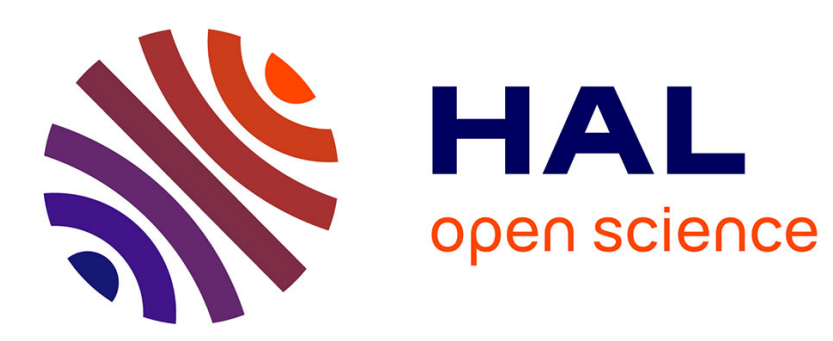

\title{
On Uniform Exponential Trichotomy of Evolution Operators in Banach Spaces
}

\author{
Mihail Megan, Codruta Stoica
}

\section{To cite this version:}

Mihail Megan, Codruta Stoica. On Uniform Exponential Trichotomy of Evolution Operators in Banach Spaces. Integral Equations and Operator Theory, 2008, 60 (4), pp.499-506. 10.1007/s00020-008-1555z . hal-00283211

\section{HAL Id: hal-00283211 \\ https://hal.science/hal-00283211}

Submitted on 29 May 2008

HAL is a multi-disciplinary open access archive for the deposit and dissemination of scientific research documents, whether they are published or not. The documents may come from teaching and research institutions in France or abroad, or from public or private research centers.
L'archive ouverte pluridisciplinaire HAL, est destinée au dépôt et à la diffusion de documents scientifiques de niveau recherche, publiés ou non, émanant des établissements d'enseignement et de recherche français ou étrangers, des laboratoires publics ou privés. 


\title{
ON UNIFORM EXPONENTIAL TRICHOTOMY OF Evolution Operators in Banach Spaces
}

\author{
Mihail Megan Codruţa Stoica
}

\begin{abstract}
This paper presents necessary and sufficient conditions for uniform exponential trichotomy of nonlinear evolution operators in Banach spaces. Thus are obtained results which extend well-known results for uniform exponential stability in the linear case.
\end{abstract}

Mathematics Subject Classification: Primary 34D05, 34D09, 93D20

Keywords: Nonlinear evolution operators, exponential stability, exponential dichotomy, exponential trichotomy

\section{Introduction}

One of the most notable results in the theory of stability of linear evolution operators has been proved by Datko in [2]. Generalizations of this result were obtained in [1], [5], [7] and [14] for exponential stability, in [9] and [1] for exponential instability and in [8], [10] and [15] for the case of exponential dichotomy.

In this paper we shall extend these results in two directions. First, we shall consider the case of uniform exponential trichotomy property ([3], [1], [6], 13]) and second, we shall not assume the linearity of evolution operators.

A unified treatment for uniform asymptotic behaviors (exponential decay, exponential growth, exponential stability, exponential instability, exponential dichotomy, exponential trichotomy) of nonlinear evolution operators is given. Examples that motivate the extension of the asymptotic behaviors for the nonlinear case are given in [5]. In our paper we obtain some theorems which extend well-known results for uniform exponential stability established in the linear case.

Let $X$ be a real or complex Banach space. The norm on $X$ will be denoted by $\|\cdot\|$. The set of all mappings from $X$ into itself is denoted by $\mathfrak{F}(X)$. Let $T$ be the set of all pairs $\left(t, t_{0}\right)$ of real numbers with the property $t \geq t_{0} \geq 0$. 


\section{Evolution operators}

Definition 2.1 A mapping $E: T \rightarrow \mathfrak{F}(X)$ is called evolution operator on $X$ if it has the property

$$
E(t, s) E\left(s, t_{0}\right)=E\left(t, t_{0}\right), \forall(t, s),\left(s, t_{0}\right) \in T .
$$

In order to emphasize the necessity of extending the study of evolution operators in the nonlinear setting, we will consider the next

Example 2.1 Let us consider the Cauchy problem

$$
\left\{\begin{array}{l}
\dot{v}(t)=A v(t), t>0 \\
v(0)=v_{0}
\end{array}\right.
$$

on a Banach space $X$ with nonlinear operator $A$. If A generates a nonlinear strongly continuous semigroup $(S(t))_{t \geq 0}$, then $E(t, s)=S(t-s)$, where $t \geq s \geq 0$, defines an evolution operator on $X$.

Definition 2.2 The evolution operator $E: T \rightarrow \mathfrak{F}(X)$ is said to be with

(i) uniform exponential decay if there exist $M>1$ and $\omega>0$ such that

$$
\left\|E\left(s, t_{0}\right) x\right\| \leq M e^{\omega(t-s)}\left\|E\left(t, t_{0}\right) x\right\|
$$

for all $t \geq s \geq t_{0} \geq 0$ and all $x \in X$;

(ii) uniform exponential growth if there are $M>1$ and $\omega>0$ such that

$$
\left\|E\left(t, t_{0}\right) x\right\| \leq M e^{\omega(t-s)}\left\|E\left(s, t_{0}\right) x\right\|
$$

for all $t \geq s \geq t_{0} \geq 0$ and all $x \in X$.

Lemma 2.1 The evolution operator $E: T \rightarrow \mathfrak{F}(X)$ has uniform exponential decay if and only if there exists a nondecreasing function $f:[0, \infty) \rightarrow(1, \infty)$ with the property

$$
\lim _{t \rightarrow \infty} f(t)=\infty
$$

such that

$$
\left\|E\left(s, t_{0}\right) x\right\| \leq f(t-s)\left\|E\left(t, t_{0}\right) x\right\|
$$

for all $t \geq s \geq t_{0} \geq 0$ and all $x \in X$.

\section{Proof.}

Necessity. It follows from Definition 2.2 (i) for $f(t)=M e^{\omega t}$.

Sufficiency. If $t \geq s \geq t_{0} \geq 0$ then there exists a natural number $n$ such that $n \leq t-s<n+1$. If we denote $M=f(1)$ and $\omega=\ln M$, then by hypothesis we have

$$
\left\|E\left(s, t_{0}\right) x\right\| \leq M\left\|E\left(s+1, t_{0}\right) x\right\| \leq M^{2}\left\|E\left(s+2, t_{0}\right) x\right\| \leq
$$




$$
\begin{aligned}
& \leq M^{n}\left\|E\left(s+n, t_{0}\right) x\right\| \leq M^{n+1}\left\|E\left(t, t_{0}\right) x\right\|= \\
& =M e^{n \omega}\left\|E\left(t, t_{0}\right) x\right\| \leq M e^{\omega(t-s)}\left\|E\left(t, t_{0}\right) x\right\|
\end{aligned}
$$

for all $t \geq s \geq t_{0} \geq 0$ and all $x \in X$.

Finally, we conclude that $E$ has exponential decay.

Lemma 2.2 The evolution operator $E: T \rightarrow \mathfrak{F}(X)$ has uniform exponential growth if and only if there exists a nondecreasing function $g:[0, \infty) \rightarrow$ $(1, \infty)$ with the property

$$
\lim _{t \rightarrow \infty} g(t)=\infty
$$

such that

$$
\left\|E\left(t, t_{0}\right) x\right\| \leq g(t-s)\left\|E\left(s, t_{0}\right) x\right\|
$$

for all $t \geq s \geq t_{0} \geq 0$ and all $x \in X$.

Proof. It is similar with the proof of Lemma 2.1.

\section{Uniform exponential trichotomy of evolution op- erators}

Let $E$ be an evolution operator on the Banach space $X$.

Definition 3.1 An application $P: \mathbb{R}_{+} \rightarrow \mathfrak{F}(X)$ is said to be a projection family on $X$ if

$$
P(t)^{2}=P(t), \forall t \in \mathbb{R}_{+} .
$$

Definition 3.2 Three projection families $P_{0}, P_{1}, P_{2}: \mathbb{R}_{+} \rightarrow \mathfrak{F}(X)$ are said to be compatible with the evolution operator $E: T \rightarrow \mathfrak{F}(X)$ if

$\left(c t_{1}\right) P_{0}(t)+P_{1}(t)+P_{2}(t)=I, \forall t \geq 0$

(ct $\left.t_{2}\right) P_{i}(t) P_{j}(t)=0, i, j \in\{0,1,2\}, i \neq j, \forall t \geq 0$

(ct $\left.t_{3}\right) E\left(t, t_{0}\right) P_{k}\left(t_{0}\right)=P_{k}(t) E\left(t, t_{0}\right), \forall\left(t, t_{0}\right) \in T$ and $k \in\{0,1,2\}$.

In what follows we will denote

$$
E_{k}\left(t, t_{0}\right)=E\left(t, t_{0}\right) P_{k}\left(t_{0}\right)=P_{k}(t) E\left(t, t_{0}\right)
$$

for all $\left(t, t_{0}\right) \in T$ and $k \in\{0,1,2\}$.

Remark 3.1 If $E$ is an evolution operator on $X$, then $E_{0}, E_{1}$ and $E_{2}$ are also evolution operators on $X$, fact proved by the following relations

$$
\begin{gathered}
E_{k}(t, s) E_{k}\left(s, t_{0}\right)=E(t, s) P_{k}(s) E\left(s, t_{0}\right) P_{k}\left(t_{0}\right)= \\
=P_{k}(t) E\left(t, t_{0}\right) P_{k}\left(t_{0}\right)=E_{k}\left(t, t_{0}\right),
\end{gathered}
$$

for all $t \geq s \geq t_{0} \geq 0$ and $k \in\{0,1,2\}$. 
Definition 3.3 An evolution operator $E: T \rightarrow \mathfrak{F}(X)$ on a Banach space $X$ is said to be uniformly exponentially trichotomic if there exist $N_{0}, N_{1}, N_{2}>$ $1, \nu_{0}, \nu_{1}, \nu_{2}>0$ and three projection families $P_{0}, P_{1}$ and $P_{2}$ compatible with E such that

$$
\begin{aligned}
& \quad\left(\text { uet }_{0}\right)\left\|E_{0}\left(s, t_{0}\right) x\right\| \leq N_{0} e^{\nu_{0}(t-s)}\left\|E_{0}\left(t, t_{0}\right) x\right\| \leq N_{0}^{2} e^{2 \nu_{0}(t-s)}\left\|E_{0}\left(s, t_{0}\right) x\right\| \\
& \quad\left(\text { uet }_{1}\right) e^{\nu_{1}(t-s)}\left\|E_{1}\left(t, t_{0}\right) x\right\| \leq N_{1}\left\|E_{1}\left(s, t_{0}\right) x\right\| \\
& \quad\left(\text { uet }_{2}\right) e^{\nu_{2}(t-s)}\left\|E_{2}\left(s, t_{0}\right) x\right\| \leq N_{2}\left\|E_{2}\left(t, t_{0}\right) x\right\| \\
& \text { for all } t \geq s \geq t_{0} \geq 0 \text { and all } x \in X \text {. }
\end{aligned}
$$

Remark 3.2 For $P_{0}=0$ we obtain the property of uniform exponential dichotomy for evolution operators studied in [8], [1]] and [15]. It is obvious that if the evolution operator $E$ is uniformly exponentially dichotomic then it is uniformly exponentially trichotomic.

Remark 3.3 If $P_{0}=P_{2}=0$ the property of uniform exponential stability is

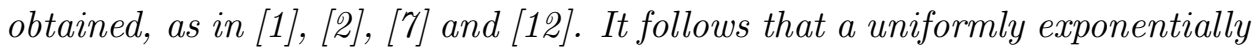
stable evolution operator is uniformly exponentially dichotomic and, further, uniformly exponentially trichotomic.

Remark 3.4 Without any loss of generality, in Definition 3.4 we can suppose that

$$
N_{0}=N_{1}=N_{2}=N \text { and } \nu_{1}=\nu_{2}=\nu
$$

because otherwise we can consider

$$
N=\max \left\{N_{0}, N_{1}, N_{2}\right\} \text { and } \nu=\min \left\{\nu_{1}, \nu_{2}\right\} .
$$

Example 3.1 Let us consider $X=\mathbb{R}^{3}$ with the norm

$$
\left\|\left(x_{1}, x_{2}, x_{3}\right)\right\|=\left|x_{1}\right|+\left|x_{2}\right|+\left|x_{3}\right|, x=\left(x_{1}, x_{2}, x_{3}\right) \in X .
$$

Let $\varphi: \mathbb{R}_{+} \rightarrow(0, \infty)$ be a decreasing continuous function with the property that there exists $\lim _{t \rightarrow \infty} \varphi(t)=l>0$.

Then the mapping $E: T \rightarrow \mathfrak{F}(X)$ defined by

$$
E\left(t, t_{0}\right) x=\left(e^{-\int_{t_{0}}^{t} \varphi(\tau) d \tau} x_{1}, e^{\int_{t_{0}}^{t} \varphi(\tau) d \tau} x_{2}, e^{-\left(t-t_{0}\right) \varphi(0)+\int_{t_{0}}^{t} \varphi(\tau) d \tau} x_{3}\right)
$$

is an evolution operator on $X$.

Let us consider the projections defined by

$$
\begin{aligned}
& P_{1}(t)\left(x_{1}, x_{2}, x_{3}\right)=\left(x_{1}, 0,0\right) \\
& P_{2}(t)\left(x_{1}, x_{2}, x_{3}\right)=\left(0, x_{2}, 0\right) \\
& P_{3}(t)\left(x_{1}, x_{2}, x_{3}\right)=\left(0,0, x_{3}\right) .
\end{aligned}
$$

for all $t \geq 0$ and all $x=\left(x_{1}, x_{2}, x_{3}\right) \in X$. 
Following relations hold

$$
\begin{gathered}
\left.\left.\| E\left(t, t_{0}\right) P_{1}\left(t_{0}\right) x\right)\left\|\leq e^{-l(t-s)}\right\| E\left(s, t_{0}\right) P_{1}\left(t_{0}\right) x\right) \| \\
\left.\left.\| E\left(t, t_{0}\right) P_{2}\left(t_{0}\right) x\right)\left\|\geq e^{l(t-s)}\right\| E\left(s, t_{0}\right) P_{2}\left(t_{0}\right) x\right) \| \\
\left.\left.\| E\left(t, t_{0}\right) P_{3}\left(t_{0}\right) x\right)\left\|\leq e^{\varphi(0)(t-s)}\right\| E\left(s, t_{0}\right) P_{3}\left(t_{0}\right) x\right) \| \\
\left.\left.\| E\left(t, t_{0}\right) P_{3}\left(t_{0}\right) x\right)\left\|\geq e^{-\varphi(0)(t-s)}\right\| E\left(s, t_{0}\right) P_{3}\left(t_{0}\right) x\right) \|
\end{gathered}
$$

for all $t \geq s \geq t_{0} \geq 0$ and all $x \in X$.

We conclude that $E$ is uniformly exponentially trichotomic.

Theorem 3.1 Let $E: T \rightarrow \mathfrak{F}(X)$ be an evolution operator on the Banach space $X$ with the property that there exist three projection families $P_{0}, P_{1}$ and $P_{2}$ compatible with $E$. Then $E$ is uniformly exponentially trichotomic if and only if there exist two nondecreasing functions $f, g:[0, \infty) \rightarrow(1, \infty)$ with the property

$$
\lim _{t \rightarrow \infty} f(t)=\lim _{t \rightarrow \infty} g(t)=\infty
$$

such that

$$
\begin{aligned}
& \left(\text { uet } t_{0}^{\prime}\right)\left\|E_{0}\left(s, t_{0}\right) x\right\| \leq f(t-s)\left\|E_{0}\left(t, t_{0}\right) x\right\| \leq f^{2}(t-s)\left\|E_{0}\left(s, t_{0}\right) x\right\| \\
& \left(\text { uet }_{1}^{\prime}\right) g(t-s)\left\|E_{1}\left(t, t_{0}\right) x\right\| \leq\left\|E_{1}\left(s, t_{0}\right) x\right\| \\
& \text { (uet } \left.{ }_{2}^{\prime}\right) g(t-s)\left\|E_{2}\left(s, t_{0}\right) x\right\| \leq\left\|E_{2}\left(t, t_{0}\right) x\right\| \\
& \text { for all } t \geq s \geq t_{0} \geq 0 \text { and all } x \in X .
\end{aligned}
$$

Proof. Necessity. As the evolution operator $E: T \rightarrow \mathfrak{F}(X)$ is uniformly exponentially trichotomic it follows from Definition 3.3 that there exist three projection families $P_{0}, P_{1}$ and $P_{2}$ compatible with $E$ such that $E_{0}$ has uniform exponential growth and uniform exponential decay, $E_{1}$ is uniformly exponentially stable and $E_{2}$ is uniformly exponentially instable.

According to Lemma 2.1 and Lemma 2.2 there exists a nondecreasing function $f:[0, \infty) \rightarrow(1, \infty)$ with the property

$$
\lim _{t \rightarrow \infty} f(t)=\infty
$$

such that

$$
\left\|E_{0}\left(s, t_{0}\right) x\right\| \leq f(t-s)\left\|E_{0}\left(t, t_{0}\right) x\right\|
$$

and

$$
\left\|E_{0}\left(t, t_{0}\right) x\right\| \leq f(t-s)\left\|E_{0}\left(s, t_{0}\right) x\right\|
$$

for all $t \geq s \geq t_{0} \geq 0$ and all $x \in X$.

Hence (uet ${ }_{0}^{\prime}$ ) is proved.

By a similar proof as in Lemma 2.1 one can characterize the properties of uniform exponential stability for $E_{1}$ and uniform exponential instability 
for $E_{2}$ (see [12]) by means of a nondecreasing function $g:[0, \infty) \rightarrow(1, \infty)$ with the property

$$
\lim _{t \rightarrow \infty} g(t)=\infty
$$

such that

$$
g(t-s)\left\|E_{1}\left(t, t_{0}\right) x\right\| \leq\left\|E_{1}\left(s, t_{0}\right) x\right\|
$$

respectively

$$
g(t-s)\left\|E_{2}\left(s, t_{0}\right) x\right\| \leq\left\|E_{2}\left(t, t_{0}\right) x\right\|
$$

for all $t \geq s \geq t_{0} \geq 0$ and all $x \in X$, which completes the proof of (uet $\left.t_{1}^{\prime}\right)$ and $\left(\right.$ uet $\left._{2}^{\prime}\right)$.

Sufficiency. According to Lemma 2.1 and Lemma 2.2, the two inequalities of statement $\left(u e t_{0}^{\prime}\right)$ imply that $E_{0}$ has exponential decay and exponential growth.

The inequality $\left(\right.$ uet $\left._{1}^{\prime}\right)$ characterize the property of uniform exponential stability for $E_{1}$ and (uet $t_{2}^{\prime}$ ) shows that $E_{2}$ is uniformly exponentially unstable, as in 12]. Thus, according to Definition 3.3, $E$ is uniformly exponentially trichotomic.

Definition 3.4 The evolution operator $E: T \rightarrow \mathfrak{F}(X)$ is said to be strongly measurable if for every $\left(t_{0}, x\right) \in \mathbb{R}_{+} \times X$ the mapping $t \rightarrow\left\|E\left(t, t_{0}\right) x\right\|$ is measurable.

Theorem 3.2 Let $E: T \rightarrow \mathfrak{F}(X)$ be an evolution operator on the Banach space $X$ with the property that there exist three projection families $P_{0}, P_{1}$ and $P_{2}$ compatible with $E$ such that the evolution operators $E_{1}$ and $E_{2}$ are strongly measurable.

Then $E$ is uniformly exponentially trichotomic if and only if

(i) $E_{0}$ and $E_{1}$ have uniform exponential growth;

(ii) $E_{0}$ and $E_{2}$ have uniform exponential decay;

(iii) there exists $M \geq 1$ such that following inequalities hold

$$
\int_{s}^{t}\left\|E_{1}\left(\tau, t_{0}\right) x\right\| d \tau \leq M\left\|E_{1}\left(s, t_{0}\right) x\right\|
$$

and

$$
\int_{s}^{t}\left\|E_{2}\left(\tau, t_{0}\right) x\right\| d \tau \leq M\left\|E_{2}\left(t, t_{0}\right) x\right\|
$$

for all $t \geq s \geq t_{0} \geq 0$ and all $x \in X$.

Proof. The property of uniform exponential trichotomy is equivalent with the existence of three projection families $P_{0}, P_{1}$ and $P_{2}$ compatible with $E$ such that $E_{0}$ is with uniform exponential growth and uniform exponential decay, $E_{1}$ is uniformly exponentially stable and $E_{2}$ is uniformly exponentially instable. 
It is sufficient to prove that if the evolution operator $E_{1}$ has uniform exponential growth and satisfies (3.2) than it is uniformly stable. Indeed, if we denote by

$$
\frac{1}{N}=\int_{0}^{1} \frac{d u}{g(u)}
$$

where function $g$ is given by Lemma 2.2, then

$$
\begin{aligned}
& \frac{\left\|E_{1}\left(t, t_{0}\right) x\right\|}{N}=\int_{t-1}^{t} \frac{\left\|E_{1}\left(t, t_{0}\right) x\right\|}{g(t-\tau)} d \tau \leq \\
& \leq \int_{s}^{t}\left\|E_{1}\left(\tau, t_{0}\right) x\right\| d \tau \leq M\left\|E_{1}\left(s, t_{0}\right) x\right\|
\end{aligned}
$$

and hence

$$
\left\|E_{1}\left(t, t_{0}\right) x\right\| \leq M N\left\|E\left(s, t_{0}\right) x\right\|
$$

for all $t \geq s+1, s \geq t_{0} \geq 0$ and all $x \in X$.

If $t \in[s, s+1]$ then

$$
\left\|E_{1}\left(t, t_{0}\right) x\right\| \leq g(t-s)\left\|E_{1}\left(s, t_{0}\right) x\right\| \leq g(1)\left\|E_{1}\left(s, t_{0}\right) x\right\|
$$

for all $s \geq t_{0} \geq 0$ and all $x \in X$.

Finally, we deduce that $E_{1}$ is uniformly exponentially stable.

Similarly, it is sufficient to prove that if the evolution operator $E_{2}$ has uniform exponential decay and satisfies relation (3.3), then it is uniformly instable.

Indeed, if we denote by

$$
\frac{1}{N}=\int_{0}^{1} \frac{d u}{f(u)}
$$

where function $f$ is given by Lemma 2.1, then

$$
\begin{aligned}
\frac{\left\|E_{2}\left(s, t_{0}\right) x\right\|}{N} & =\int_{s}^{s+1} \frac{\left\|E_{2}\left(s, t_{0}\right) x\right\|}{f(v-s)} d v \leq \int_{s}^{s+1}\left\|E_{2}\left(v, t_{0}\right) x\right\| d v \leq \\
& \leq \int_{t_{0}}^{t}\left\|E_{2}\left(v, t_{0}\right) x\right\| d v \leq M\left\|E_{2}\left(t, t_{0}\right) x\right\|
\end{aligned}
$$

and hence

$$
\left\|E_{2}\left(s, t_{0}\right) x\right\| \leq M N\left\|E_{2}\left(t, t_{0}\right) x\right\|
$$

for all $t \geq s \geq t_{0} \geq 0$ and all $x \in X$ and so $E_{2}$ is uniformly exponentially instable.

Remark 3.5 Theorem 3.9 can be considered a generalization of a wellknown result due to Datko (Theorem 11 from [2]). We remark that our proofs are not generalizations of Datko's proof for the characterization of the uniform exponential stability property. 


\title{
References
}

[1] C. Buşe, On nonuniform exponential stability of evolutionary processes. Rend. Sem. Mat. Univ. Polit. Torino 52 (1994), 395-406.

[2] R. Datko, Uniform asymptotic stability of evolutionary processes in Banach spaces. SIAM J. Math. Anal. 3 (1972), 428-445.

[3] S. Elaydi, O. Hajek, Exponential trichotomy of differential systems. J. Math. Anal. Appl. 129 (1988), 362-374.

[4] S. Elaydi, O. Hajek, Exponential dichotomy and trichotomy of nonlinear differential equations. Diff. Integral. Eqs. 3 (1990), 1201-1224.

[5] A. Ichikawa, Equivalence of $L_{p}$ stability for a class of nonlinear semigroups. Nonlinear Analysis 8 (1984) No. 7, 805-815.

[6] H. Jianlin, Exponential trichotomies and Fredholm operators. Ann. of Diff. Equations 9 (1993), 37-43.

[7] M. Megan, On (h,k)-stability of evolution operators in Banach spaces. Dynamic Systems and Applications 5 (1996), 189-196.

[8] M. Megan, D.R. Laţcu, Exponential dichotomy of evolution operators in Banach spaces. Internat. Ser. Num. Math, Birkhäuser Verlag 107 (1992), 47-62.

[9] M. Megan, A.L. Sasu, B. Sasu, Banach function spaces and exponential instability of evolution operators. Archivum Mathematicum 39 (2003), 277-286.

[10] M. Megan, A.L. Sasu, B. Sasu, On nonuniform exponential dichotomy of evolution operators in Banach spaces. Integral Equations Operator Theory 44 (2002), 71-78.

[11] M. Megan, A. Pogan, On exponential h-expansiveness of semigroups of operators in Banach spaces. Nonlinear Analysis 52 (2003), 545-556.

[12] M. Megan, C. Stoica, On asymptotic behaviors of evolution operators in Banach spaces. Seminar on Mathematical Analysis and Applications in Control Theory, West University of Timisoara (2006), 1-22.

[13] M. Megan, C. Stoica, On null uniform exponential trichotomy of evolution operators in Hilbert spaces. Annals of the Tiberiu Popoviciu Seminar on Functional Equations, Approximation and Convexity 3 (2005), 141-150.

[14] J.M.A.M. van Neerven, The asymptotic behavior of linear operators. Birkhäuser Verlag, 1996.

[15] P. Preda, M. Megan, Exponential dichotomy of evolutionary processes in Banach spaces. Czechoslovak Mathematical Journal 35 (1985) No. 110, 312-323.

\author{
Minail Megan \\ Faculty of Mathematics \\ West University of Timişoara \\ Bd. Pârvan, No.4 \\ 300223 Timişoara \\ Romania \\ E-mail: megan@math.uvt.ro \\ Codruţa Stoica \\ Department of Mathematics \\ Aurel Vlaicu University of Arad \\ Bd. Revoluţiei, No. 77 \\ 310130 Arad \\ Romania \\ E-mail: stoicad@rdslink.ro
}

